\title{
Enzymes Related to Polychlorinated Biphenyl and Enzyme Kinetics
}

\section{Xinqing Yang*}

Institute of Biotechnology, Shanxi University, China

Polychlorinated biphenyls (PCBs) are now one of the most serious recalcitrant pollutants, the use of microorganisms is expected to be an effective tool for remediation of PCB-polluted environments. In the most PCB degrader, the conversion of biphenyl to a benzoic and other 2-hydroxypenta-2,4-dienoate is catalyzed by a series of enzymes: biphenyl dioxygease (BphA), dihydrodia dehydroxygenase (BphB), 2,3-dihydroxybiphenyl dioxygenase (BphC) and 2-hydroxyl6-oxo-6-phenylhexa-2,4-dienoic acid hydrolase (BphD). Biphenyl 2,3-dioxygenase catalyzes the oxygenation of two vicinal ortho-meta carbons of the biphenyl ring to yield a 2,3-dihydrodihydroxybiphenyl (DDBP). The dihydrodiol intermediate is then substrate for BphB, an $\mathrm{NAD}+$ dependent dehydrogenase that produces 2,3-dihydroxybiphenyl (DHBP) for BphC, a ring fission dioxygenase. Following ring fission, hydrolytic cleavage by $\mathrm{BphD}$ produces benzoate and 2-hydroxypenta2,4-dienoate.

In Rhodococcus sp. strain R04, genes (bphBCA1A2A3A4D) encoding for biphenyl 2,3-dioxygenase (BphA1A2A3A4), cis-2,3-dihydro-2,3dihydroxybiphenyl dehydrogenase (BphB), 2,3-dihydroxybiphenyl 1,2-dioxygenase (BphC) and 2-hydroxy-6-oxo-6-phenylhexa-2,4dienoate hydrolase (HOPDA hydrolase, BphD), played an important role in degradation of biphenyl, and organized in an operon. The $b p h$ genes in strain R04 are preceded by $b p h B$, and followed by $b p h C$, $b p h A 1 A 2 A 3 A 4$ and $b p h D$. The gene encoding the $\alpha$-subunit of biphenyl dioxygenase (BphA1) separated from bphC by an orf 2 of unknown function. Furthermore, a $330 \mathrm{bp}$ and a $450 \mathrm{bp}$ fragments are localized between $b p h A 2$ and $b p h A 3, b p h A 3$ and $b p h A 4$, respectively. A more detailed analysis revealed that $b p h$ genes organization in strain R04 varied from other PCB degrader.

We have characterized two enzymes BphC and BphD in the $b p h$ pathway, and proved them to be thermostable. Pre-steady-state analysis was carried out on wild-type $\mathrm{BphD}$ and the data simulated by curve-fitting. The results shows that Ser-110 plays a dominant role in the course of $\mathrm{C}-\mathrm{C}$ cleavage; His-265 is responsible for ketonisation of the substrate and participates $\mathrm{C}-\mathrm{C}$ cleavage along with Ser-110 and Trp-266; in the course of C-C cleavage catalyzed by the BphD, Trp266 also plays a very important role in this enzyme catalyzed reaction expect for catalytic triad (Ser-110, Asp-237, His-265).

Our objective is to provide the reader with some view of the 2,3-dihydroxybiphenyl 1,2-dioxygenase and biphenyl hydrolase, including (a) the regulation of PCB metabolism to several 2,3-dihydroxybiphenyl 1,2-dioxygenase, (b) the directed evolution of biphenyl hydrolase, (c) pre-steady-stable kinetics of biphenyl hydrolase and its mutants. We hope that the result of this endeavour is a repository of information that will be useful not only for the specialist to delve the wishes into the complexities of a specific area but also for those more casual readers just wanting to dip their toes in the water.
*Corresponding author: Xinqing Yang, Institute of Biotechnology, Shanxi University, China, E-mail: xiuqyang@sxu.edu.cn

Received October 18, 2012; Accepted October 20, 2012; Published October 22 2012

Citation: Yang X (2012) Enzymes Related to Polychlorinated Biphenyl and Enzyme Kinetics. Biochem Anal Biochem 1:e124. doi:10.4172/2161-1009.1000e124

Copyright: () 2012 Yang X. This is an open-access article distributed under the terms of the Creative Commons Attribution License, which permits unrestricted use, distribution, and reproduction in any medium, provided the original author and source are credited. 